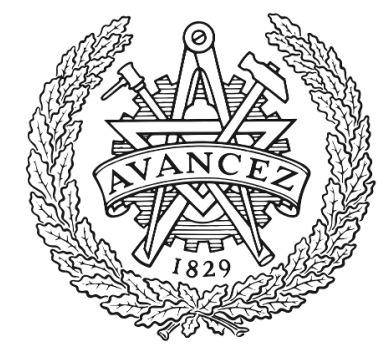

CHALMERS

UNIVERSITY OF TECHNOLOGY

\title{
Tensor Decomposition-based Beamspace Esprit Algorithm for Multidimensional Harmonic Retrieval
}

Downloaded from: https://research.chalmers.se, 2023-04-26 15:03 UTC

Citation for the original published paper (version of record):

Wen, F., So, H., Wymeersch, H. (2020). Tensor Decomposition-based Beamspace Esprit Algorithm for Multidimensional Harmonic Retrieval. ICASSP, IEEE International Conference on Acoustics, Speech and Signal Processing - Proceedings, 2020-May: 4572-4576.

http://dx.doi.org/10.1109/ICASSP40776.2020.9053619

N.B. When citing this work, cite the original published paper. 


\title{
TENSOR DECOMPOSITION-BASED BEAMSPACE ESPRIT ALGORITHM FOR MULTIDIMENSIONAL HARMONIC RETRIEVAL
}

\author{
Fuxi Wen*, Hing Cheung So ${ }^{\dagger}$, Henk Wymeersch* \\ *Department of Electrical Engineering, Chalmers University of Technology, Sweden \\ ${ }^{\dagger}$ Department of Electrical Engineering, City University of Hong Kong, Hong Kong, China
}

\begin{abstract}
Beamspace processing is an efficient and commonly used approach in harmonic retrieval (HR). In the beamspace, measurements are obtained by linearly transforming the sensing data, thereby achieving a compromise between estimation accuracy and system complexity. Meanwhile, the widespread use of multi-sensor technology in HR has highlighted the necessity to move from a matrix (two-way) to tensor (multiway) analysis. In this paper, we propose a beamspace tensorESPRIT for multidimensional HR. In our algorithm, parameter estimation and association are achieved simultaneously.
\end{abstract}

Index Terms - Tensor, beamspace-ESPRIT, harmonic retrieval, CANDECOMP/PARAFAC decomposition.

\section{INTRODUCTION}

Multidimensional ( $R$-D) harmonic retrieval (HR) is required in numerous signal processing problems [1] and has been studied for many decades in a variety of fields, such as mobile communications [2], multiple-input multiple-output (MIMO) radar [3] and nuclear magnetic resonance spectroscopy [4]. A number of HR techniques are available in the literature [5]. Estimation of signal parameters via rotational invariance techniques (ESPRIT) [6] and its variants [7, 8] have become one of the popular search-free signal subspacebased parameter estimation techniques [9]. A tensor is a natural approach to describe $R$-D data for $R \geq 3$ [10]. CANDECOMP/PARAFAC (CP) and Tucker are two widely used tensor decomposition approaches [11]. Note that association of frequencies in each dimension is also important for multidimensional HR. In tensor-ESPRIT-type algorithms, the association is usually achieved by computing the eigenvalues of the estimated matrices jointly via a joint approximate eigen-decomposition [12] or a simultaneous Schur decomposition [13].

In some applications HR must be performed in beamspace, due to hardware constraints or achieving a compromise between estimation accuracy and system complexity. An im-

This project has received funding from the European Union's Horizon 2020 under the Marie Skłodowska-Curie grant agreement No. 700044 and the Swedish Research Council under grant No. 2018-03701.

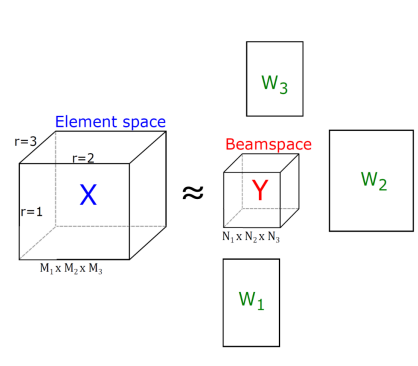

(a)

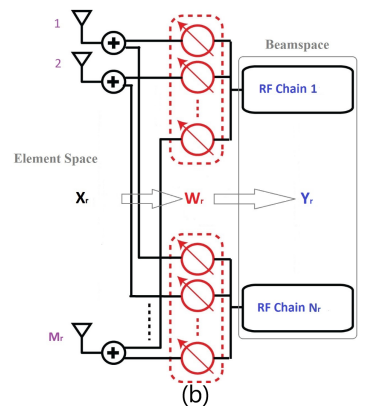

Fig. 1. Illustration of measurements in beamspace. (a) general case. (b) MIMO example with hybrid combining.

portant application is millimeter wave (mmWave) MIMO communications, where beamspace measurements naturally occur due to hybrid array architectures [14]. In beamspace $\mathrm{HR}$, the sensing data are not available directly, but measurements are obtained by their linear transformation. Correspondingly, they are called beamspace measurements to distinguish between element space measurements in standard HR. As shown in Fig. 1, element space measurements of dimensions $M_{1} \times M_{2} \times M_{3}$ are linearly transformed to obtain beamspace measurements of dimensions $N_{1} \times N_{2} \times N_{3}$. Beamspace ESPRIT methods were proposed in [15] and recently applied to mmWave MIMO communications [16] for $R=3$. A similar problem was addressed in [17], where a tensor decomposition-aided mmWave channel estimation algorithm was developed for $R=3$ in a discrete Fourier transform (DFT) beamspace. However, the method requires a line search for each of the dimensions, so that the complexity may be too high for real-time applications [5].

A search-free $R$-D and general beamspace tensor-ESPRIT algorithm is proposed in [18] for mmWave MIMO channel estimation. In this paper, we extend the algorithm in [18] for multidimensional HR. We first evaluate the bias and root-mean square error (RMSE) performance for different beamspaces, followed by demonstrating the robustness of the method with respect to uncertainty in the number of sources, as well as the applicability for sources with partially distinct frequencies. 


\section{PROBLEM FORMULATION}

We use $(\cdot)^{\mathrm{H}},(\cdot)^{\mathrm{T}}$ and $\mathbb{C}$ to denote Hermitian transpose, transpose and the set of complex numbers, respectively. We follow the tensor operations defined in [19]. The $\left(i_{1}, i_{2}, \cdots, i_{R}\right)$ entry of an $R$-D tensor $\mathcal{A}$ is denoted as $a_{i_{1}, i_{2}, \cdots, i_{R}}$.

Element-Space Model We consider sequential transmissions, where for the $k$ th snapshot, the element-space tensor $\mathcal{X}_{k}$ has entries of the form: ${ }^{1}$

$$
x_{m_{1}, \cdots, m_{R}, k}=\sum_{l=1}^{L} \gamma_{l}(k) \prod_{r=1}^{R} e^{j \omega_{r, l} \times m_{r}},
$$

where $m_{r}=0,1, \cdots, M_{r}-1, r=1,2, \cdots, R, l=$ $1,2, \cdots, L$. Here, $M_{r}, R$ and $L$ denote the number of sensors for the $r$ th dimension, the number of dimensions and the number of $R$-D frequencies (i.e., number of signal paths or sources), respectively, $\gamma_{l}(k)$ represents the unknown complex amplitude of the $l$ th frequency at the $k$ th snapshot, while $\omega_{r, l} \in(-\pi, \pi)$ is the frequency in the $r$ th dimension of the $l$ th source. For multiple snapshots, the tensor dimension is $(R+1)$. After obtaining the $R L$ frequencies, parameter association is required to obtain the structured information of the measurements. The frequencies associated to the $l$ th source are denoted as $\left\{\omega_{1, l}, \omega_{2, l}, \cdots, \omega_{R, l}\right\}$, where, $l=1,2, \cdots, L$. The number of sources $L$ is assumed to be known (e.g., from an $R$-D source enumeration method [20]). The tensor $\mathcal{X}_{k} \in \mathbb{C}^{M_{1} \times M_{2} \times \cdots \times M_{R}}$ can be expressed as

$$
\mathcal{X}_{k}=\sum_{l=1}^{L} \gamma_{l} \mathbf{a}_{1, l} \circ \mathbf{a}_{2, l} \circ \cdots \circ \mathbf{a}_{R, l},
$$

where $\mathbf{a}_{r, l}=\left[\begin{array}{llll}e^{j \omega_{r, l}} & e^{j 2 \omega_{r, l}} & \cdots & e^{j M_{r} \omega_{r, l}}\end{array}\right]^{\top}$ and $\circ$ denotes the vector outer product [11]. We also define:

$$
\mathbf{A}_{r}=\left[\begin{array}{llll}
\mathbf{a}_{r, 1} & \mathbf{a}_{r, 2} & \cdots & \mathbf{a}_{r, L}
\end{array}\right] \in \mathbb{C}^{M_{r} \times L} .
$$

Beamspace Model For beamspace measurements, after the $r$-mode product of $\mathcal{X}_{k}$ with linear transformation matrix [11], the model (2) is modified to

$$
\mathcal{Y}_{k}=\sum_{l=1}^{L} \gamma_{l} \mathbf{b}_{1, l} \circ \mathbf{b}_{2, l} \circ \cdots \circ \mathbf{b}_{R, l},
$$

where the beamspace array manifold is defined as

$$
\mathbf{B}_{r}=\left[\begin{array}{llll}
\mathbf{b}_{r, 1} & \mathbf{b}_{r, 2} & \cdots & \mathbf{b}_{r, L}
\end{array}\right]=\mathbf{W}_{r}^{\mathrm{H}} \mathbf{A}_{r} \in \mathbb{C}^{N_{r} \times L} .
$$

Here $\mathbf{W}_{r}^{\mathrm{H}}=\left[\begin{array}{llll}\mathbf{w}_{r, 1} & \mathbf{w}_{r, 2} & \cdots & \mathbf{w}_{r, M_{r}}\end{array}\right] \in \mathbb{C}^{N_{r} \times M_{r}}$ is the linear transformation matrix. The transformation (5) can be interpreted as a matrix beamformer. The columns of transformation matrix $\mathbf{W}_{r}$ are usually chosen as beamformers to

\footnotetext{
${ }^{1}$ For notational compactness, noise is omitted in the equations
}

cover a sector of source locations, provided that a priori information about the true frequencies is available. The DFT beamforming matrix is one of the common transformation matrices, which is defined as

$$
\mathbf{W}_{r}=\left[\begin{array}{llll}
\mathbf{a}_{r, 1} & \mathbf{a}_{r, 2} & \cdots & \mathbf{a}_{r, N_{r}}
\end{array}\right] \in \mathbb{C}^{M_{r} \times N_{r}},
$$

where the pointing frequencies $\omega_{r, n_{r}}, n_{r}=1,2, \cdots, N_{r}$, are spaced uniformly within the sector of interest [21]. The number $N_{r}$ should be chosen properly, so that the beams cover most of the signal energy. Furthermore, $\mathbf{W}_{r}^{\mathrm{H}} \mathbf{W}_{r}=\mathbf{I}_{N_{r}}$ is required to maintain white in the beamspace output. This is achievable by whitening the non-orthogonal beams [22].

Our objective is to estimate $\omega_{r, l}$, for $r=1, \cdots, R$ and $l=1, \cdots, L$, from noisy measurements $\tilde{\mathcal{Y}}_{k}=\mathcal{Y}_{k}+\mathcal{V}_{k}$, using the beamspace tensor-ESPRIT method and $\mathcal{V}_{k}$ denotes the white Gaussian noise tensor.

\section{3. $R$-D BEAMSPACE TENSOR-ESPRIT FOR HR}

In the CP decomposition, a tensor is decomposed into a sum of rank-one component tensors,

$$
\tilde{\mathcal{Y}}_{k}=\sum_{l=1}^{L} \lambda_{l} \mathbf{u}_{1, l} \circ \mathbf{u}_{2, l} \circ \cdots \circ \mathbf{u}_{R, l} .
$$

Both association and noise reduction are achieved simultaneously. ESPRIT algorithms utilize the shift invariant property:

$$
\mathbf{J}_{r}^{(1)} \mathbf{A}_{r}=\mathbf{J}_{r}^{(2)} \mathbf{A}_{r} \boldsymbol{\Phi}_{r},
$$

where $\boldsymbol{\Phi}_{r}$ contains the frequencies of all sources in $r$ th dimension, $\boldsymbol{\Phi}_{r}=\operatorname{diag}\left[\begin{array}{llll}e^{-j \omega_{r, 1}} & e^{-j \omega_{r, 2}} & \cdots & e^{-j \omega_{r, L}}\end{array}\right]$, $\mathbf{J}_{r}^{(1)}=\left[\begin{array}{ll}\mathbf{I}_{N_{r}-1} & \mathbf{0}_{\left(N_{r}-1\right) \times 1}\end{array}\right]$ and $\mathbf{J}_{r}^{(2)}=\left[\begin{array}{ll}\mathbf{0}_{\left(N_{r}-1\right) \times 1} & \mathbf{I}_{N_{r}-1}\end{array}\right]$ are selection matrices.

In beamspace, the row transformation $\mathbf{W}_{r}^{\mathrm{H}}$ alters the transitional invariance structure in the array manifold [15], and consequently $\mathbf{J}_{r}^{(1)} \mathbf{B}_{r} \neq \mathbf{J}_{r}^{(2)} \mathbf{B}_{r} \boldsymbol{\Phi}_{r}$. However, the shift invariance structure can be restored, if $\mathbf{W}_{r}$ has a similar structure. Suppose we are able to find a non-singular $N_{r} \times N_{r}$ matrix $\mathbf{F}_{r}$ that satisfies

$$
\mathbf{J}_{r}^{(1)} \mathbf{W}_{r}=\mathbf{J}_{r}^{(2)} \mathbf{W}_{r} \mathbf{F}_{r}
$$

The least squares (LS) estimate of $\mathbf{F}_{r}$ is given by

$$
\hat{\mathbf{F}}_{r}=\left(\mathbf{J}_{r}^{(2)} \mathbf{W}_{r}\right)^{\dagger} \mathbf{J}_{r}^{(1)} \mathbf{W}_{r}
$$

where $\dagger$ denotes the pseudo-inverse.

Theorem 1. Let $\mathbf{F}_{r}$ be defined as in (9). If there exists a $\mathbf{Q}_{r} \in \mathbb{C}^{N_{r} \times N_{r}}$, such that

$$
\mathbf{Q}_{r} \mathbf{w}_{r, M_{r}}=\mathbf{0}_{N_{r} \times 1} \text {, and } \mathbf{Q}_{r} \mathbf{F}_{r}^{\mathrm{H}} \mathbf{w}_{r, 1}=\mathbf{0}_{N_{r} \times 1},
$$

then

$$
\mathbf{Q}_{r} \mathbf{F}_{r}^{\mathrm{H}} \mathbf{B}_{r}=\mathbf{Q}_{r} \mathbf{B}_{r} \boldsymbol{\Phi}_{r}^{\mathrm{H}}
$$


Proof. See Appendix A in [18].

It is worth mentioning that $\mathbf{Q}_{r}$ in (11) can be obtained by forming a projection matrix corresponding to the orthogonal subspace of $\mathcal{R}\left\{\mathbf{w}_{r, M_{r}}, \mathbf{F}_{r}^{\mathrm{H}} \mathbf{w}_{r, 1}\right\}$. Then

$$
\hat{\mathbf{Q}}_{r}=\mathbf{I}_{N_{r}}-\mathbf{w}_{r, M_{r}} \mathbf{w}_{r, M_{r}}^{\mathrm{H}}-\left(\mathbf{F}_{r}^{\mathrm{H}} \mathbf{w}_{r, 1}\right)\left(\mathbf{F}_{r}^{\mathrm{H}} \mathbf{w}_{r, 1}\right)^{\mathrm{H}} .
$$

Let $\mathbf{U}_{r}=\left[\begin{array}{llll}\mathbf{u}_{r, 1} & \mathbf{u}_{r, 2} & \cdots & \mathbf{u}_{r, L}\end{array}\right]$, its columns span the signal subspace, and replace $\mathbf{B}_{r}$ by $\mathbf{B}_{r}=\mathbf{U}_{r} \mathbf{D}_{r}$, where $\mathbf{D}_{r} \in \mathbb{C}^{L \times L}$ is a non-singular matrix. Then (12) becomes

$$
\mathbf{Q}_{r} \mathbf{F}_{r}^{\mathrm{H}} \mathbf{U}_{r}=\mathbf{Q}_{r} \mathbf{U}_{r} \boldsymbol{\Gamma}_{r}
$$

where

$$
\boldsymbol{\Gamma}_{r}=\mathbf{D}_{r} \boldsymbol{\Phi}_{r}^{\mathrm{H}} \mathbf{D}_{r}^{-1} \in \mathbb{C}^{L \times L} .
$$

It is interesting to note that $\mathbf{D}_{r}$ is incorporated in $\boldsymbol{\Gamma}_{r}$, direct computing is not required. We estimate $\boldsymbol{\Gamma}_{r}$ via LS from (14):

$$
\hat{\boldsymbol{\Gamma}}_{r}=\left(\mathbf{Q}_{r} \mathbf{U}_{r}\right)^{\dagger} \mathbf{Q}_{r} \mathbf{F}_{r}^{\mathrm{H}} \mathbf{U}_{r} .
$$

From (15), eigenvalues of $\boldsymbol{\Gamma}_{r}$ are the diagonal elements of $\boldsymbol{\Phi}_{r}^{\mathrm{H}}$, so that the $l$ th eigenvalue of $\boldsymbol{\Gamma}_{r}$ is given by $e^{j \omega_{r, l}}$. Note that the $l$ th column of $\mathbf{U}_{r}$ corresponds to the same source. For the $r$ th dimension, the $L$ frequencies $\boldsymbol{\omega}_{r}=$ $\left\{\omega_{r, 1}, \omega_{r, 2}, \cdots, \omega_{r, L}\right\}$ can be estimated jointly from the eigenvalues of $\boldsymbol{\Gamma}_{r}$. The proposed algorithm is summarized in Algorithm 1.

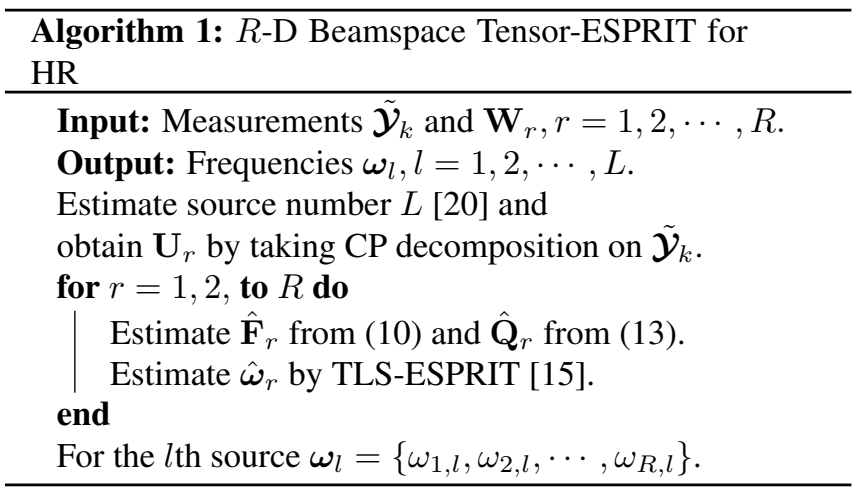

\section{SIMULATION RESULTS}

The performance of the proposed method is evaluated in terms of the bias and RMSE on estimated parameters:

$$
\text { Bias }=\frac{1}{R L} \sum_{r=1}^{R} \sum_{l=1}^{L}\left(\omega_{r, l}-\hat{\omega}_{r, l}\right)
$$

and RMSE $=\sqrt{\frac{1}{R L} \mathbb{E}_{t}\left\{\sum_{r=1}^{R} \sum_{l=1}^{L}\left(\omega_{r, l}-\hat{\omega}_{r, l}\right)^{2}\right\}}$, where $\hat{\omega}_{r, l}$ is an estimate of $\omega_{r, l}$, and $\mathbb{E}_{t}\{\cdot\}$ denotes the expected value based on 200 Monte-Carlo trials. The proposed method is compared with tensor-ESPRIT (T-ESPRIT) algorithm [7] in element space, as well as Cramér-Rao lower bound (CRLB). In the following simulations, a DFT beamforming matrix is considered. The unknown amplitude of the $l$ th frequency at the $k$ th snapshot, $\gamma_{l}(k)$, is drawn from $\mathcal{N}(0,1)$. Signal-to-noise ratio (SNR) is defined as SNR $=$ $\left\|\tilde{\mathcal{X}}_{k}-\mathcal{V}_{k}\right\|_{F}^{2} /\left\|\mathcal{V}_{k}\right\|_{F}^{2}$, where $\|\cdot\|_{F}$ denotes the tensor Frobenius norm [11]. In element space, $M_{1}=M_{2}=M_{3}=8$ and the number of measurements is $K=10$. The Matlab package Tensorlab [23] is utilized for tensor computation. In the first test, there are three sources and the frequencies to be estimated are:

$$
\begin{aligned}
& r=1:\left(\omega_{1,1}, \omega_{1,2}, \omega_{1,3}\right)=(0.550 \pi, 0.719 \pi, 0.906 \pi) \\
& r=2:\left(\omega_{2,1}, \omega_{2,2}, \omega_{2,3}\right)=(0.410 \pi, 0.777 \pi, 0.276 \pi) \\
& r=3:\left(\omega_{3,1}, \omega_{3,2}, \omega_{3,3}\right)=(0.340 \pi, 0.906 \pi, 0.358 \pi) .
\end{aligned}
$$

Fig. 2 shows the 3-D HR parameter estimation performance versus SNR using DFT beamforming matrix. RMSE performance under different SNR is shown in Fig. 2. Comparison element space T-ESPRIT is also included. For beamspace ESPRIT, the data dimensions are $N_{1} \times N_{2} \times N_{3}$, and different values of $N_{r}$ are considered: $N_{r}=4,6$ and 8 . We observe that both bias and RMSE are reduced by increasing the size of the matrix beamformer. The performance is close to element space ESPRIT when a larger $N_{r}$ is used.

Sources with partially distinct frequencies are common phenomena in real applications. In the second test, the performance of the proposed method for partially distinct frequencies is evaluated. Now there are four sources, and the frequencies are same in at least one of the 3 dimensions. In the beamspace, $N_{1}=N_{2}=N_{3}=6$ and and SNR is $20 \mathrm{~dB}$. The 3-D HR frequencies to be estimated are:

$$
\begin{aligned}
& r=1:\left(\omega_{1,1}, \omega_{1,2}, \omega_{1,3}, \omega_{1,4}\right)=(0.2 \pi, 0.2 \pi, 0.6 \pi, 0.8 \pi) \\
& r=2:\left(\omega_{2,1}, \omega_{2,2}, \omega_{2,3}, \omega_{2,4}\right)=(0.9 \pi, 0.4 \pi, 0.4 \pi, 0.6 \pi) \\
& r=3:\left(\omega_{3,1}, \omega_{3,2}, \omega_{3,3}, \omega_{3,4}\right)=(0.1 \pi, 0.2 \pi, 0.8 \pi, 0.8 \pi) .
\end{aligned}
$$

As shown in Fig. 3, the four sources are estimated and associated correctly, even with partially distinct frequencies.

In the third test, different source numbers are considered. In the beamspace, $N_{1}=N_{2}=N_{3}=6$ and SNR is $10 \mathrm{~dB}$. The 3-D HR frequencies to be estimated are:

$$
\begin{aligned}
& r=1:\left(\omega_{1,1}, \omega_{1,2}, \omega_{1,3}\right)=(0.1 \pi, 0.3 \pi, 0.8 \pi) \\
& r=2:\left(\omega_{2,1}, \omega_{2,2}, \omega_{2,3}\right)=(0.9 \pi, 0.4 \pi, 0.2 \pi) \\
& r=3:\left(\omega_{3,1}, \omega_{3,2}, \omega_{3,3}\right)=(0.4 \pi, 0.1 \pi, 0.7 \pi) .
\end{aligned}
$$

The number of sources is $L=3$. The amplitudes of the first, second and third sources are $1,0.85$ and 0.75 , respectively. As shown in Fig. 4, the 3-D parameters are associated, even with inaccurate source number information. Let $\hat{L}$ be 


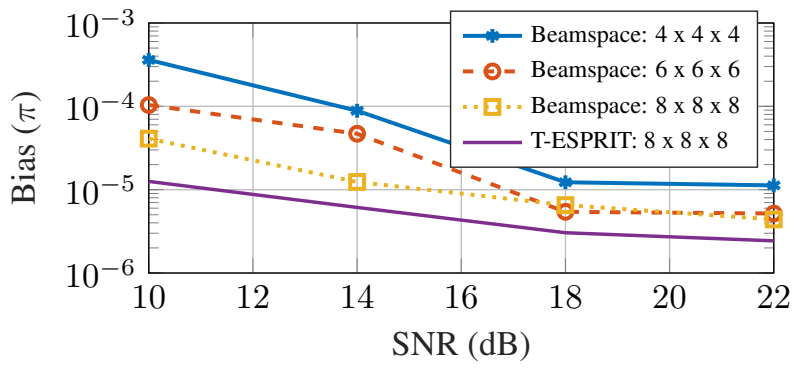

(a) Bias.

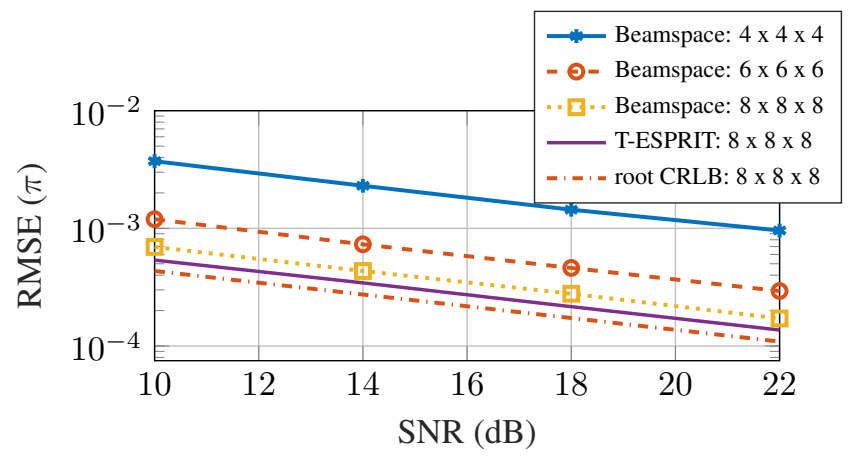

(b) RMSE.

Fig. 2. Parameter estimation performance versus SNR for distinct frequencies.

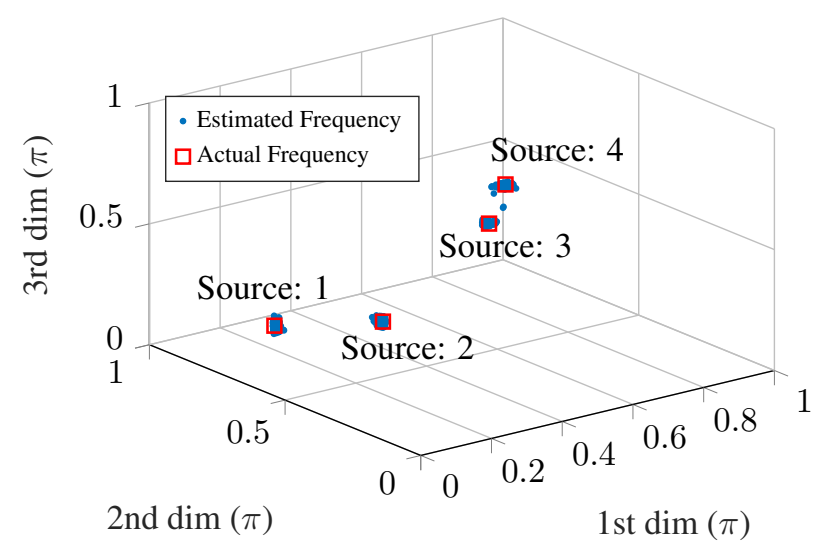

Fig. 3. Parameter estimation for partially distinct frequencies. the estimated number of sources. An interesting observation is that, if $\hat{L} \leq L$, then the first dominant $\hat{L}$ sources are identified, and the corresponding results are shown in Fig. 4(a)-Fig. 4(c). Otherwise, the first dominant $L$ sources are still observable, but the extra fake sources are randomly distributed, as shown in Fig. 4(d).

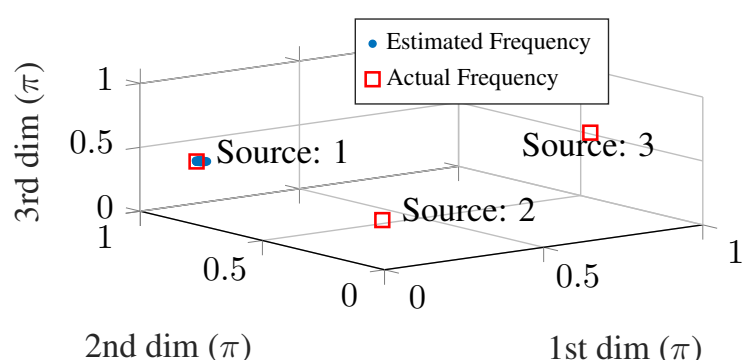

(a) Estimated number of sources $\hat{L}=1$.

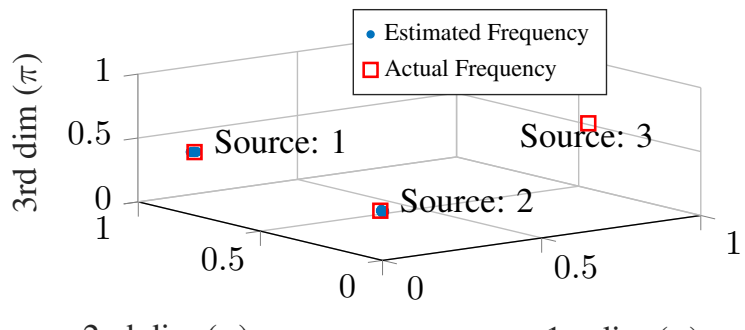

2nd $\operatorname{dim}(\pi) \quad 1$ st $\operatorname{dim}(\pi)$

(b) Estimated number of sources $\hat{L}=2$.

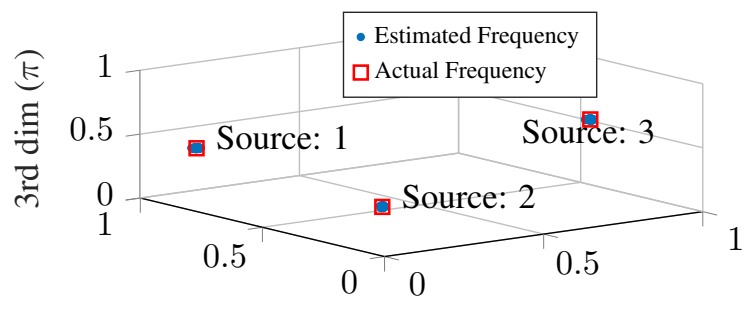

2nd $\operatorname{dim}(\pi) \quad 1$ st $\operatorname{dim}(\pi)$

(c) Estimated number of sources $\hat{L}=3$.

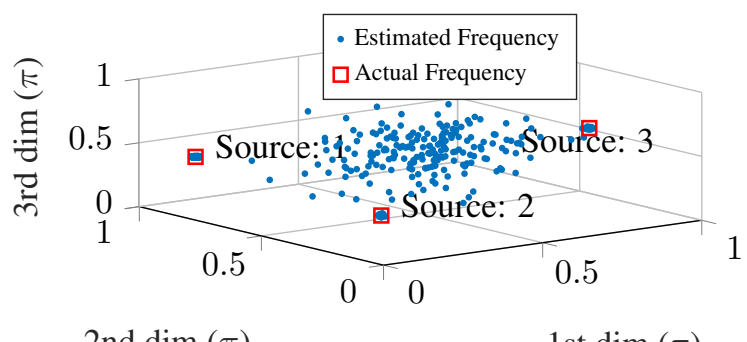

2nd $\operatorname{dim}(\pi) \quad 1$ st $\operatorname{dim}(\pi)$

(d) Estimated number of sources $\hat{L}=4$.

Fig. 4. Parameter association performance for the proposed method with inaccurate source number information. 


\section{CONCLUSION}

A beamspace $R$-D tensor-ESPRIT algorithm is developed for multidimensional harmonic retrieval. Source parameters estimation and association are achieved simultaneously. Furthermore, the effect of errors in the estimated number of sources is investigated, as well as the applicability for sources with partially distinct frequencies is demonstrated.

\section{REFERENCES}

[1] X. Liu, N. D. Sidiropoulos, and T. Jiang, Space-Time Processing for MIMO Communications. NY: Wiley, 2005, ch. 2. Multidimensional harmonic retrieval with applications in MIMO wireless channel sounding, pp. $41-75$.

[2] M. Pesavento, C. F. Mecklenbräuker, and J. F. Böhme, "Multidimensional rank reduction estimator for parametric MIMO channel models," EURASIP Journal on Advances in Signal Processing, vol. 2004, no. 9, pp. 1354-1363, 2004.

[3] D. Nion and N. Sidiropoulos, "Tensor algebra and multidimensional harmonic retrieval in signal processing for MIMO radar," IEEE Transactions on Signal Processing, vol. 58, no. 11, pp. 5693-5705, Nov. 2010.

[4] Y. Li, J. Razavilar, and K. J. R. Liu, "A high-resolution technique for multidimensional NMR spectroscopy," IEEE Transactions on Biomedical Engineering, vol. 45, no. 1, pp. 78-86, Jan. 1998.

[5] A. B. Gershman, M. Rübsamen, and M. Pesavento, "One- and two-dimensional direction-of-arrival estimation: An overview of search-free techniques," Signal Processing, vol. 90, no. 5, pp. 1338-1349, 2010.

[6] R. Roy and T. Kailath, "ESPRIT-estimation of signal parameters via rotational invariance techniques," IEEE Transactions on Acoustics, Speech, and Signal Processing, vol. 37, no. 7, pp. 984-995, Jul. 1989.

[7] M. Haardt, F. Roemer, and G. Del Galdo, "Higher-order SVD-based subspace estimation to improve the parameter estimation accuracy in multidimensional harmonic retrieval problems," IEEE Transactions on Signal Processing, vol. 56, no. 7, pp. 3198-3213, Jul. 2008.

[8] F. Roemer, M. Haardt, and G. D. Galdo, "Analytical performance assessment of multi-dimensional matrix- and tensor-based ESPRIT-type algorithms," IEEE Transactions on Signal Processing, vol. 62, no. 10, pp. 26112625, May 2014.

[9] M. Haardt, M. Pesavento, F. Roemer, and M. N. El Korso, "Subspace methods and exploitation of special array structures," in Academic Press Library in Signal Processing: Vol. 3 Array and Statistical Signal Processing, ser. Electronic Reference in Signal Processing, A. M. Zoubir, M. Viberg, R. Chellappa, and S. Theodoridis, Eds. Elsevier, Jan. 2014, pp. 651-717.

[10] A. Cichocki, D. Mandic, L. De Lathauwer, G. Zhou, Q. Zhao, C. Caiafa, and H. A. Phan, "Tensor decompositions for signal processing applications: From two-way to multiway component analysis," IEEE Signal Processing Magazine, vol. 32, no. 2, pp. 145-163, Mar. 2015.

[11] T. G. Kolda and B. W. Bader, "Tensor decompositions and applications," SIAM Review, vol. 51, no. 3, pp. 455500, Aug. 2009.

[12] T. Fu and X. Gao, "Simultaneous diagonalization with similarity transformation for non-defective matrices," in Proc. IEEE International Conference on Acoustics Speech and Signal Processing, Toulouse, France, May 2006, pp. 1137-1140.

[13] M. Haardt and J. A. Nossek, "Simultaneous Schur decomposition of several nonsymmetric matrices to achieve automatic pairing in multidimensional harmonic retrieval problems," IEEE Transactions on Signal Processing, vol. 46, no. 1, pp. 161-169, Jan. 1998.

[14] R. W. Heath, N. González-Prelcic, S. Rangan, W. Roh, and A. M. Sayeed, "An overview of signal processing techniques for millimeter wave MIMO systems," IEEE Journal of Selected Topics in Signal Processing, vol. 10, no. 3, pp. 436-453, Apr. 2016.

[15] G. Xu, S. D. Silverstein, R. H. Roy, and T. Kailath, "Beamspace ESPRIT," IEEE Transactions on Signal Processing, vol. 42, no. 2, pp. 349-356, Feb. 1994.

[16] J. Zhang and M. Haardt, "Channel estimation and training design for hybrid multi-carrier mmWave massive MIMO systems: The beamspace ESPRIT approach," in Proc. 25th European Signal Processing Conference, Kos, Greece, Aug. 2017, pp. 385-389.

[17] Z. Zhou, J. Fang, L. Yang, H. Li, Z. Chen, and R. S. Blum, "Low-rank tensor decomposition-aided channel estimation for millimeter wave MIMO-OFDM systems," IEEE Journal on Selected Areas in Communications, vol. 35, no. 7, pp. 1524-1538, Jul. 2017.

[18] F. Wen, N. Garcia, J. Kulmer, K. Witrisal, and H. Wymeersch, "Tensor decomposition based beamspace ESPRIT for millimeter wave MIMO channel estimation," in IEEE Global Communications Conference, Dec. 2018, pp. 1-7. 
[19] L. De Lathauwer, B. De Moor, and J. Vandewalle, "A multilinear singular value decomposition," SIAM Journal on Matrix Analysis and Applications, vol. 21, no. 4, pp. 1253-1278, 2000.

[20] K. Liu, J. P. C. da Costa, H. C. So, L. Huang, and J. Ye, "Detection of number of components in CANDECOMP/PARAFAC models via minimum description length," Digital Signal Processing, vol. 51, pp. 110 123, 2016.

[21] M. D. Zoltowski, G. M. Kautz, and S. D. Silverstein, "Beamspace root-MUSIC," IEEE Transactions on Signal Processing, vol. 41, no. 1, pp. 344-364, Jan. 1993.

[22] P. Stoica and A. Nehorai, "Comparative performance study of element-space and beam-space MUSIC estimators," Circuits, Systems and Signal Processing, vol. 10, no. 3, pp. 285-292, Sep. 1991.

[23] N. Vervliet, O. Debals, L. Sorber, M. Van Barel, and L. De Lathauwer. (2016, Mar.) Tensorlab 3.0. [Online]. Available: https://www.tensorlab.net 